\title{
Constraining composite Higgs models with direct and indirect searches
}

\section{Christoph Niehoff*†}

Excellence Cluster Universe, TUM, Boltzmannstr. 2, 85748 Garching, Germany

E-mail: christoph.niehoffeph.tum.de

In this talk I present a comprehensive numerical analysis of a model with a composite pseudoNambu-Goldstone-Higgs arising from the minimal coset $\mathrm{SO}(5) / \mathrm{SO}(4)$. For this all relevant experimental constraints stemming from direct and indirect searches as well as from a realistic electroweak symmetry breaking are taken into account. Concrete predictions for flavour observables are given and the prospects for experimental searches are investigated.

38th International Conference on High Energy Physics 3-10 August 2016

Chicago, USA

\footnotetext{
* Speaker.

${ }^{\dagger}$ In collaboration with Peter Stangl and David M. Straub
} 


\section{Introduction}

Although its huge success in describing the experimental data the Standard Model (SM) leaves a few open questions unanswered. In particular, it does not give any explanation of why the mass of the Higgs boson is relatively small compared to any New Physics (NP) scale. Furthermore, flavour mixings and hierarchies appear as input parameters and no explanation for their structure is given.

These problems can be lifted if one assumes that the Higgs boson is a composite bound state of some new strong interactions, which takes the role of a pseudo-Nambu-Goldstone boson (pNGB) of a broken global symmetry in this new sector. In this case, the mass of the Higgs boson is generated by the interactions of the strongly interacting composite sector with SM-like elementary states that break the global symmetries explicitely. Additionally, assuming some well-defined flavour structure for the composite states the SM flavour patterns are explained also by the interactions between composite and elementary sector.

This talk is based on [1]. Here we present a comprehensive numerical analysis of a concrete model of the above class, which is realistic from the point of view of model building as well as it is phenomenologically successfull in being compatible with all current experimental constraints. To be specific, we want to constrain the parameter space of a bottom-up EFT parametrization for a composite Higgs model, where we include direct constraints (from searches at colliders), indirect constraints (from precision observables and flavour transitions) as well as a realistic electroweak symmetry breaking that reproduces the correct Higgs mass and the vacuum expectation value (vev).

\section{Model}

The theory space for the above construction is large. In this work, the selection of a concrete model is guided by the fact that the model should be minimal in its assumptions and parameters but also successful in passing all phenomenological tests. This leads to the following considerations.

- The protection of electroweak precision observables (most notably the $S$ and $T$ parameters) calls for some kind of custodial symmetry $\mathrm{SU}(2)_{\mathrm{L}} \times \mathrm{SU}(2)_{\mathrm{R}} \cong \mathrm{SO}(4)$ [2].

- At the LHC no extension of the scalar sector was observed so far. Therefore, we stick to the minimal coset of global symmetry breaking $\mathrm{SO}(5) / \mathrm{SO}(4)$ that features 4 Goldstone fields corresponding to a single complex Higgs doublet.

- Under these global symmetries the composite fermion sector can be organized in different representations. We choose the minimal possibility of fundamentals 5 under $\mathrm{SO}(5)$ that allow for a custodial protection of $Z b_{\mathrm{L}} b_{\mathrm{L}}$ couplings which are experimentally very constrained [3].

- Minimality in the number of parameters enforces a two-site description in which the SM is supplemented by only one level of composite resonances.

- Of particular interest for us is a Higgs potential that is able to reproduce the correct Higgs mass and its vev. In this type of models the Higgs potential is generated dynamically by the Coleman-Weinberg mechanism. Thus, a-priori it is not given that the potential is finite and its calculability has to be ensured by either enforcing Weinberg Sum Rules [4] or by working 
in a framework in which the composite structure is deconstructed from a higher-dimensional gauge theory, such that the potential is protected by gauge invariance. We choose the second option as it allows to further reduce the number of free parameters.

- As we are mostly interested in quark flavour phenomenology, we assume the lepton sector to the trivially elementary such that no new parameters are introduced here. However, composite leptons can in principle lead to interesting phenomenological effects [5, 6].

Putting all these conditions together, we chose to implement the two-sited Minimial 4D Composite Higgs Model [7] with the minimal coset $\mathrm{SO}(5) / \mathrm{SO}(5)$ and fermion resonances embedded in the fundamental representation. To account for flavour transitions we assume that the strong sector is invariant under a flavour symmetry $G_{\mathrm{F}}=\mathrm{U}(3)^{3}$ or $\mathrm{U}(2)^{3}$ that is only broken by either the left-handed (right-compositeness, RC) or right-handed (left-compositeness, LC) [8, 9] interactions between the elementary and composite sectors.

\section{Numerical Analysis and experimental Constraints}

The goal of this work is to find parameter points $\vec{\theta}$ that are compatible with all current experimental constraints. To this end, we construct a $\chi^{2}$ function,

$$
\chi^{2}(\vec{\theta}) \equiv \sum_{i, j \in \text { observables }}\left(\mathscr{O}_{i}^{\mathrm{th}}(\vec{\theta})-\mathscr{O}_{i}^{\exp }\right)\left[C_{\text {total }}^{2}\right]_{i j}^{-1}\left(\mathscr{O}_{j}^{\mathrm{th}}(\vec{\theta})-\mathscr{O}_{j}^{\exp }\right)
$$

such that the problem reduces to finding the minima of $\chi^{2}(\vec{\theta})$ on the space of all parameters. Unfortunately, the dimensionality of the parameter space for the above realistic Composite Higgs model is high $(\mathscr{O}(40))$ and due to partial compositeness all calculable observables are complicated functions of all model parameters allowing no simple extraction of the allowed ranges for a few parameters alone. As a consequence we are forced to use rather involved numerical techniques, where in particular we use Markov Chain Monte Carlos to sample the parameter space [10].

In the analysis we include the following constraints:

- The most apparent constraint is reproducing the SM particle masses, CKM mixings and the correct vev. For this we first calculate the Higgs potential and determine its minima. Depending on this, mass mixing matrices of the combined composite and elementary sector are diagonalized, giving masses that we compare to the SM masses at the scale $\mu=m_{t}$. RGE running above $m_{t}$ we neglect, however. CKM elements are calculated through tree-level $W$-vertices giving a non-unitary CKM matrix that strongly constrains the quark degrees of compositeness.

- Due to custodial symmetry there are no tree-level contributions to the $T$-parameter. At oneloop level we include fermion contributions. The $S$-parameter, however, already gets contributions $S \sim 1 / m_{\rho}^{2}$ at tree-level that effectively act as a lower bound on vector resonance masses.

- Corrections to couplings of the $Z$ boson to SM fermions give an important bound on the degrees of compositeness [11]. In this model the couplings to left-handed down-type quarks are custodially protected. 
- The flavour structure is constrained by including meson-antimeson-mixing in the $B_{d}, B_{s}$ and $K$ systems. We also include rare $B$ decays such as $B_{s} \rightarrow \mu^{+} \mu^{-}$and the dipole transition $b \rightarrow s \gamma$.

- As electroweak symmetry is realized non-linearly in the Higgs sector corrections on Higgs production and decay have to be included since they give important constraints on the New Physics scale $f$. To be concrete, we include the signal strengths $h \rightarrow W W, Z Z, \tau \tau$ at tree-level as well as $h \rightarrow g g, \gamma \gamma$ at loop-level.

- If the light quarks have one chirality with a large degree of compositeness, contact interactions arise. These we include as constraints on the dijet angular distribution at the LHC [12].

- As a last and very important point we include direct searches for new particles at colliders. These give constraints on the masses and branching ratios for heavy vector resonances and vector-like quarks. We implement all relevant channels coming from LEP, Tevatron and the LHC.

\section{Results}

The main result of this analysis is a large collection of parameter points that fulfill the experimental constraints and have a reasonable coverage of all relevant areas of parameter space.
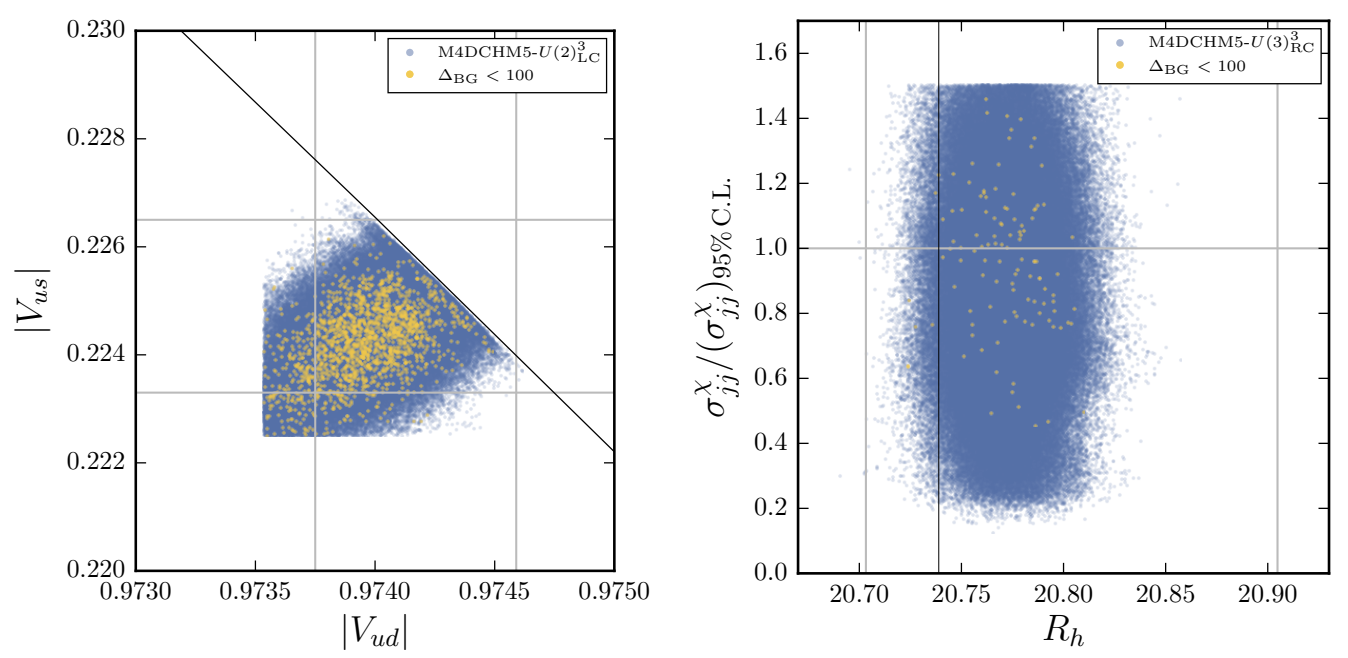

Figure 1: Scatter plots for left- and right-compositeness. Blue point corresponds to one parameter point that fulfills all experimental constraints at the $3 \sigma$-level. The gray lines denote the $2 \sigma$ experimental bounds. The $3 \sigma$ condition is visible as vertical and horizontal cuts in the parameter point distribution. By the yellow dots we denote points with good finetuning. left: The main bound on left-compositeness is given by CKM unitarity. right: For right-compositeness the dijet angular distributions are most constraining, while e.g. the hadronic $Z$ width does not place any significant bound.

Depending on the chosen model (i.e. on the assumed flavour symmetry) one can identify the most constraining bounds. As shown in fig. 1 the strongest constraint on left-compositeness comes 
from CKM unitarity which is clear as for this case the flavour structure leads to a significant degree of compositeness of the left-handed SM quarks. For right-compositeness this is not the case and here the contact interactions give the strongest bound on the compositeness of light quarks.

A consequence of this is that the bounds of CKM unitarity are particularly strong for the case of a $\mathrm{U}(3)^{3}$ flavour symmetry with left-compositeness as here the degree of compositeness of the light quarks is directly linked to the compositeness of the left-handed top quark. In fact, we do not find any viable parameter points for this flavour structure such that we can exclude this scenario.
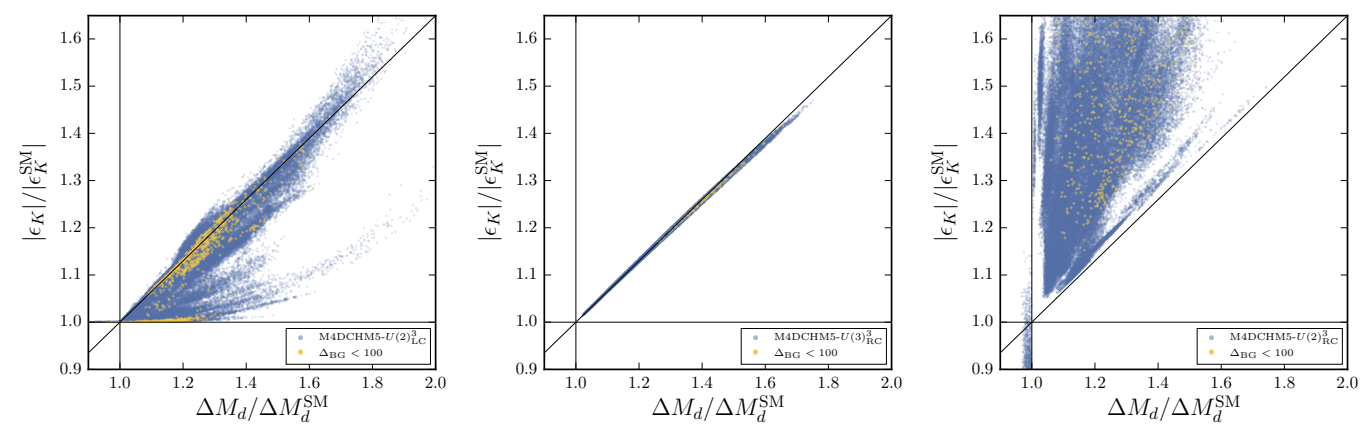

Figure 2: Correlations between flavour observables for all three viable flavour structures. We present the mass difference in the $B_{d}$ system $\Delta M_{d}$ and the indirect $C P$ violation $\varepsilon_{K}$ in the Kaon system, each normalized to the corresponding $\mathrm{SM}$ value. left: $\mathrm{U}(2)_{\mathrm{LC}}^{3}$, middle: $\mathrm{U}(3)_{\mathrm{RC}}^{3}$, right: $\mathrm{U}(2)_{\mathrm{RC}}^{3}$.

In flavour observables we find that large effects (up to saturating the experimental bounds) are possible e.g. in meson-antimeson mixing. Including also observables for $C P$ violation, the assumed flavour symmetries lead to distinct patterns. As shown in fig. 2 these pattern can be used to distinguish between the different flavour scenarios.

For all three flavour structures we find that a Barbieri-Giudice measure of finetuning [13] well below 100 is possible, showing that this type of models features a finetuning similar to other NP models.
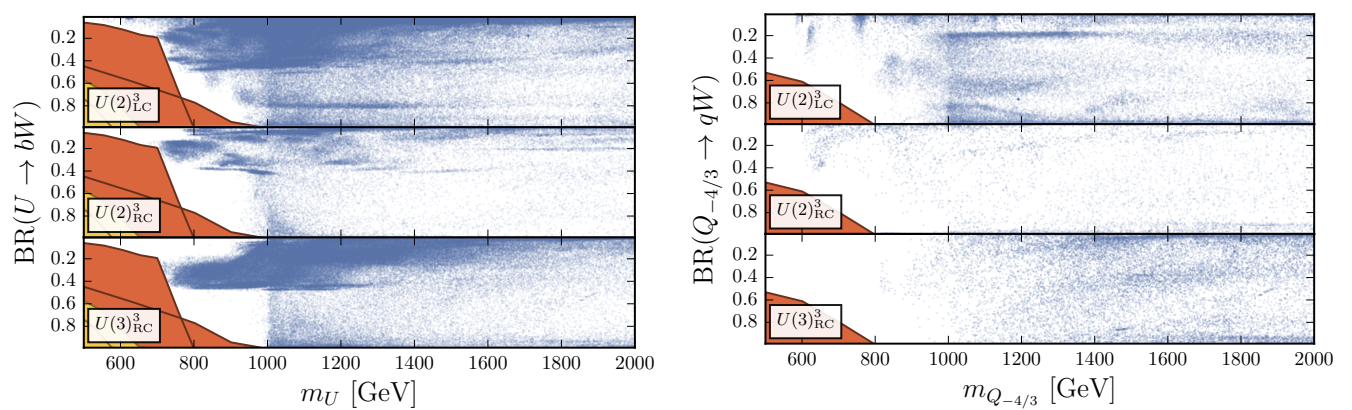

Figure 3: Prospects for different experimental channels to observe a signal for the considered model. The orange and yellow areas denote the regions in the mass - branching ratio plane that are excluded by LHCrun1 data. The blue dots show the theoretical expectations in this model for all three viable flavour structures. left: The channel of a heavy top partner decaying into $b W$ is very promising. right: In the channel of a heavy vector-like quark with exotic charge $-\frac{4}{3}$ decaying into $W+$ jet the current experimental bounds are rather weak. 
For the valid parameter points one can compare their collider phenomenology, which allows to identify the experimental channels that are most promissing to observe a signal or cut deeply into the parameter space of this model. In fig. 3 we show examples for this.

\section{Conclusion}

In this talk I presented a comprehensive numerical analysis of a model with a pNGB-Higgs arising from the minimal coset $\mathrm{SO}(5) / \mathrm{SO}(4)$ where I take into account all relevant experimental constraints stemming from direct and indirect searches as well as from a realistic electroweak symmetry breaking. From this concrete predictions are given for flavour observables and prospects for experimental searches are shown. Furthermore, a flavour symmetry $\mathrm{U}(3)_{\mathrm{LC}}^{3}$ is excluded.

For further details I refer to the original publication [1].

\section{Acknowledgements}

I want to thank the organizers of ICHEP2016 for giving me the opportunity to present these results in the beautiful city of Chicago. Special thanks go to Peter Stangl and David M. Straub for this collaboration. This research was supported by the DFG cluster of excellence "Origin and Structure of the Universe".

\section{References}

[1] C. Niehoff, P. Stangl, and D. M. Straub, Direct and indirect signals of natural composite Higgs models, JHEP 01 (2016) 119, [arXiv: 1508 .00569].

[2] K. Agashe, R. Contino, and A. Pomarol, The Minimal composite Higgs model, Nucl. Phys. B719 (2005) 165-187, [hep-ph/ 0412089 ].

[3] K. Agashe, R. Contino, L. Da Rold, and A. Pomarol, A Custodial symmetry for Zbb̆, Phys. Lett. B641 (2006) 62-66, [hep-ph/ 0605341$].$

[4] D. Marzocca, M. Serone, and J. Shu, General Composite Higgs Models, JHEP 08 (2012) 013, [arXiv:1205.0770].

[5] A. Carmona and F. Goertz, A naturally light Higgs without light Top Partners, JHEP 05 (2015) 002, [arXiv: 1410.8555$]$.

[6] C. Niehoff, P. Stangl, and D. M. Straub, Violation of lepton flavour universality in composite Higgs models, Phys. Lett. B747 (2015) 182-186, [arXiv:1503.03865].

[7] S. De Curtis, M. Redi, and A. Tesi, The 4D Composite Higgs, JHEP 04 (2012) 042, [arXiv:1110.1613].

[8] G. Cacciapaglia, C. Csaki, J. Galloway, G. Marandella, J. Terning, and A. Weiler, A GIM Mechanism from Extra Dimensions, JHEP 04 (2008) 006, [arXiv: 0709.1714 ].

[9] M. Redi and A. Weiler, Flavor and CP Invariant Composite Higgs Models, JHEP 11 (2011) 108, [arXiv:1106.6357].

[10] F. Beaujean and S. Jahn, pypmc version 1.0, feb, 2015. DOI:10.5281/zenodo.20045. 
[11] D. M. Straub, Anatomy of flavour-changing Z couplings in models with partial compositeness, JHEP $\mathbf{0 8}(2013)$ 108, [arXiv:1302.4651].

[12] M. de Vries, Four-quark effective operators at hadron colliders, JHEP 03 (2015) 095, [arXiv:1409.4657].

[13] R. Barbieri and G. F. Giudice, Upper Bounds on Supersymmetric Particle Masses, Nucl. Phys. B306 (1988) 63-76. 